\title{
High-Stability, High-Efficiency Organic Monoliths Made of Oligomer Nanoparticles Wrapped in Organic Matrix
}

Zeliha Soran-Erdem, ${ }^{\dagger \dagger}$ Talha Erdem, ${ }^{\dagger,}$ Kivanc Gungor, ${ }^{\dagger}$ Jousheed Pennakalathil, $^{\dagger}$ Dönüs Tuncel, and Hilmi Volkan Demir ${ }^{*},, \|$

${ }^{\dagger}$ Departments of Electrical and Electronics Engineering, Physics, and Chemistry, UNAM-National Nanotechnology Research Center, and Institute of Materials Science and Nanotechnology, Bilkent University, Ankara 06800, Turkey

"Luminous! Center of Excellence for Semiconductor Lighting and Displays, School of Electrical and Electronic Engineering, School of Physical and Mathematical Sciences, Nanyang Technological University, Singapore 639798, Singapore

\section{Supporting Information}

ABSTRACT: Oligomer nanoparticles (OL NPs) have been considered unsuitable for solid-state lighting due to their low quantum yields and low temperature stability of their emission. Here, we address these problems by forming highly emissive and stable OL NPs solids to make them applicable in lighting. For this purpose, we incorporated OL NPs into sucrose matrix and then prepared their all-organic monoliths. We show that wrapping the OL NPs in sucrose significantly increases their quantum yield up to $44 \%$, while the efficiency of their dispersion and direct solid-film remain only at $\sim 6 \%$. We further showed $\sim 3$-fold improved temperature stability of OL NP emission within these monoliths. Our experiments revealed that a physical passivation mechanism is responsible from these improvements. As a proofof-concept demonstration, we successfully employed these high-stability, high-efficiency monoliths as color converters on a blue LED chip.

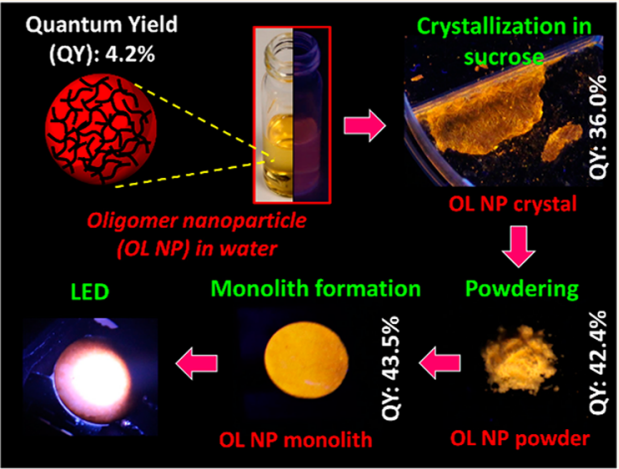
Considering the improved optical features, low cost, and simplicity of the presented methodology, we believe that this study holds great promise for a ubiquitous use of organic OL NPs in lighting and possibly in other photonic applications.

KEYWORDS: oligomer nanoparticles, fluorescent monoliths, organic crystalline host, light-emitting diodes, solid-state lighting

onjugated polymers, which gain their semiconducting features from the delocalization of $\pi$-electrons along the polymer chain, ${ }^{1}$ have attracted significant interest for numerous applications including light-emitting diodes (LEDs), ${ }^{2-5}$ solar cells, ${ }^{6-8}$ bioimaging,,${ }^{9,10}$ and sensing. ${ }^{11}$ Their easy processability is the main factor making them highly attractive for the aforementioned applications. ${ }^{12}$ Additionally, the structure of used monomers allows for tuning their absorption and emission spectra. Attaching various side groups further increases their functionality in terms of the applications. ${ }^{1}$ A key acquisition of these conjugated polymers is the capability of their nanoparticle formation resulting in increased dispersibility in water, low toxicity, and reduced sensitivity of the optical features to environmental changes such as thin film formation. ${ }^{13}$ However, the problem of significant decrease in the quantum yield (QY) after solution to nanoparticle (NP) and film transition, which is attributed to the aggregate-induced depopulation of the excitons, ${ }^{14-17}$ still persists and strongly restricts the use of conjugated polymers in solid film.
Recently, conjugated oligomer-based nanoparticles have emerged as an alternative to the conjugated polymer nanoparticles. ${ }^{18}$ Oligomer (OL) nanoparticles composed of smaller molecules with well-defined molecular weight compared to conjugated polymer nanoparticles allow for faster guest release, which is especially attractive for drug delivery applications. Furthermore, Fischer et al. reported a higher fluorescent quantum yield with comparable stability and molar absorptivity in comparison with conjugated polymer nanoparticles. ${ }^{19}$ However, the related literature is still scarce despite the potential of this class of organic nanoparticles. Among the few studies is the work of Dang et al., which reports the synthesis and characterization of copper phthalocyanine oligomer nanoparticles. $^{20}$ In another study, Liu et al. employed conjugated oligomers to functionalize gold nanoparticles and studied their self-assembly as spherical particles. ${ }^{21}$ And recently,

Received: February 29, 2016

Accepted: May 5, 2016

Published: May 5, 2016 

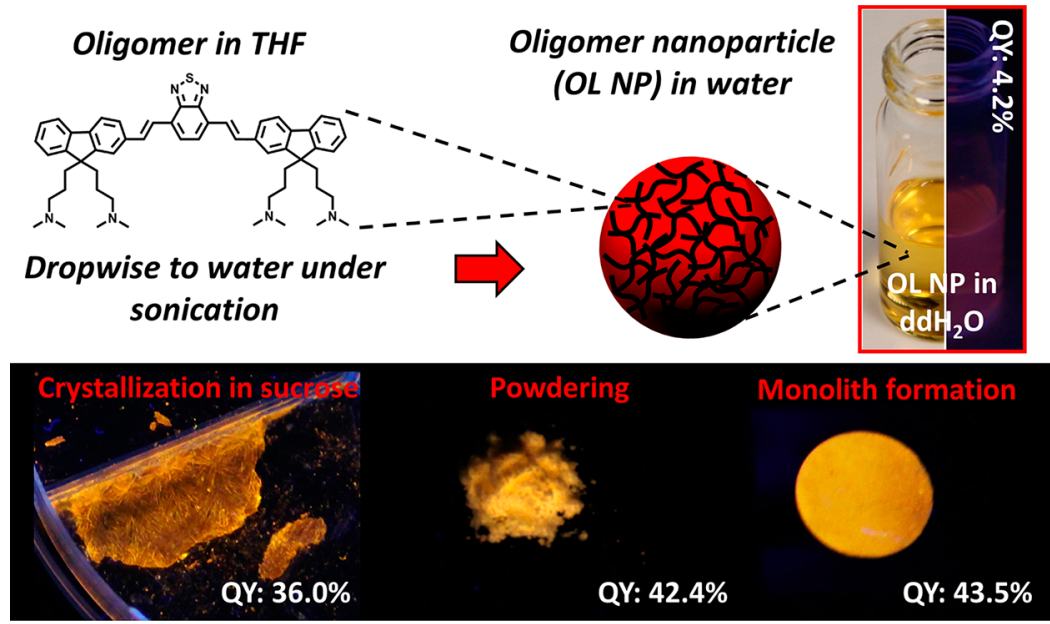

Figure 1. Schematic diagram depicting the preparation of oligomer nanoparticles and the real color images of OL NP in dispersion, crystal, powder, and monolith form, along with their quantum yields.
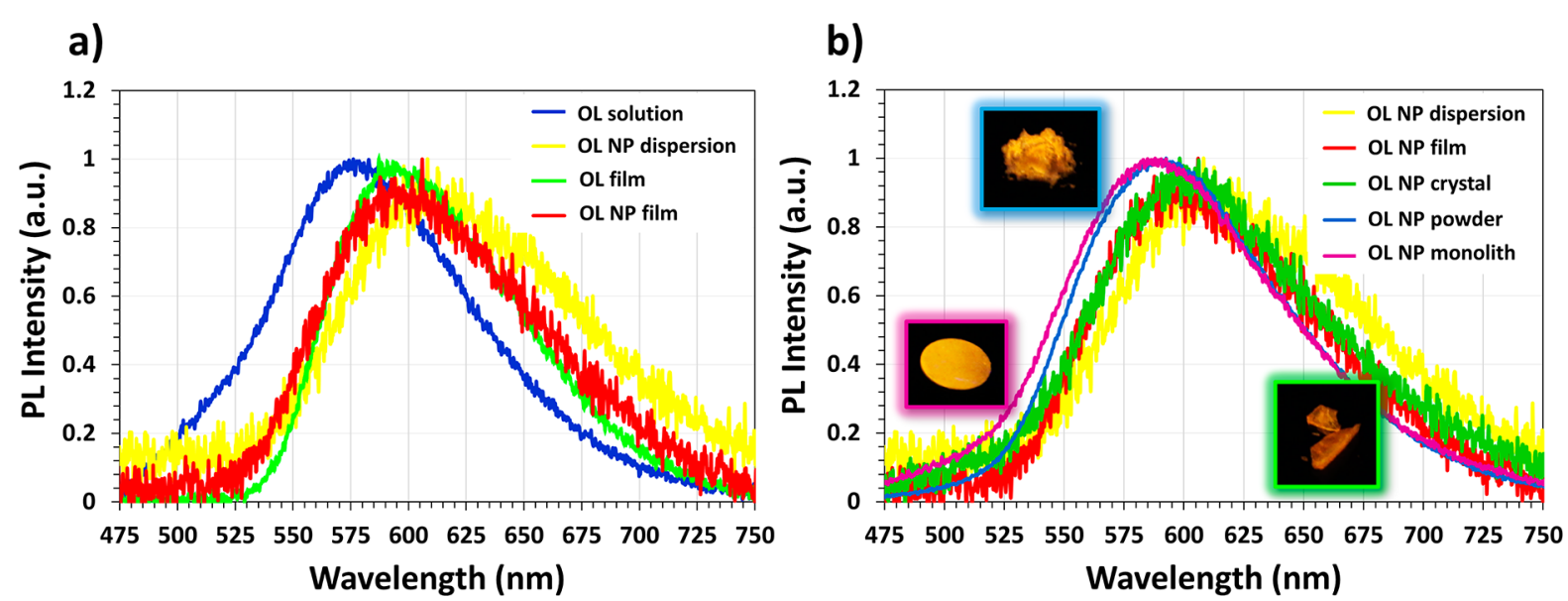

Figure 2. (a) Photoluminescence spectra belonging to OL solution in THF, OL NP dispersion in water, and their solid films. (b) Photoluminescence intensity of OL NP dispersion, solid-film and OL NP crystal, powder, and monolith. Insets show the real-color images of OL NP crystal, powder, and monolith.

we demonstrated the use of $\mathrm{pH}$-sensitive orange-emitting conjugated oligomer nanoparticles as drug delivery agents on MCF7 and MDA-MB-231 breast cancer cell lines. ${ }^{18}$

Nevertheless, these OL NPs could not yet be successfully implemented for solid-state lighting application. The main reason behind this has been the inability to obtain strongly emitting color-converting solid films. For example, spin-coating technique forms only very thin films, limiting the applicability of the nanoparticles as color converters on LEDs. Drop-casting, on the other hand, allows for the formation of thick films that might be suitable for color conversion; however, in this case, the resulting low quantum yields associated with the aggregation remain as the main bottleneck. In addition to these, their emission stability at elevated temperatures is prohibitively low. This problem has to be addressed for possible deployment in industrial use. This is because the light-emitting diode chips driven at high currents that optically excite the nanoparticles expose the nanoparticles to high temperatures. Previously, inorganic semiconductor nanocrystals were investigated using a salt matrix, ${ }^{2-24}$ for which our group studied similar stability issues. ${ }^{25,26}$ However, to date OL NPs have never been investigated to remedy the critical problems of low quantum yield and low temperature stability. Currently, there exists no materials solution to make high-stability, highefficiency oligomer nanoparticles in solid form.

To address these challenges and achieve highly stable and emissive organic solids applicable to solid-state lighting, here we show the incorporation of oligomer nanoparticles into a host crystalline matrix and formation of high-efficiency and highstability all-organic monoliths from their powders. We observed that immobilizing these nanoparticles within a salt matrix does not help to improve their QYs. However, wrapping them in an appropriate organic crystal (in our case, sucrose) increases the QY to $\sim 7.5$ times compared to their direct solid film without using a crystal host, also accompanied by a $\sim 3$-fold improved temperature stability.

This approach brings the performance of the oligomer nanoparticles in solid state to comparable levels with that of inorganic emitters. We subsequently investigated the emission dynamics of the OL NPs integrated into the host crystal sucrose and attributed the efficiency increase to possible reversible noncovalent interactions between the organic nanoparticles and sucrose lattice. The achieved thickness, size, QY, and stability of the OL NP integrated sucrose monoliths make the oligomer nanoparticles suitable for solid-state lighting applications. With this motivation, we demonstrated a proof-of- 
a)
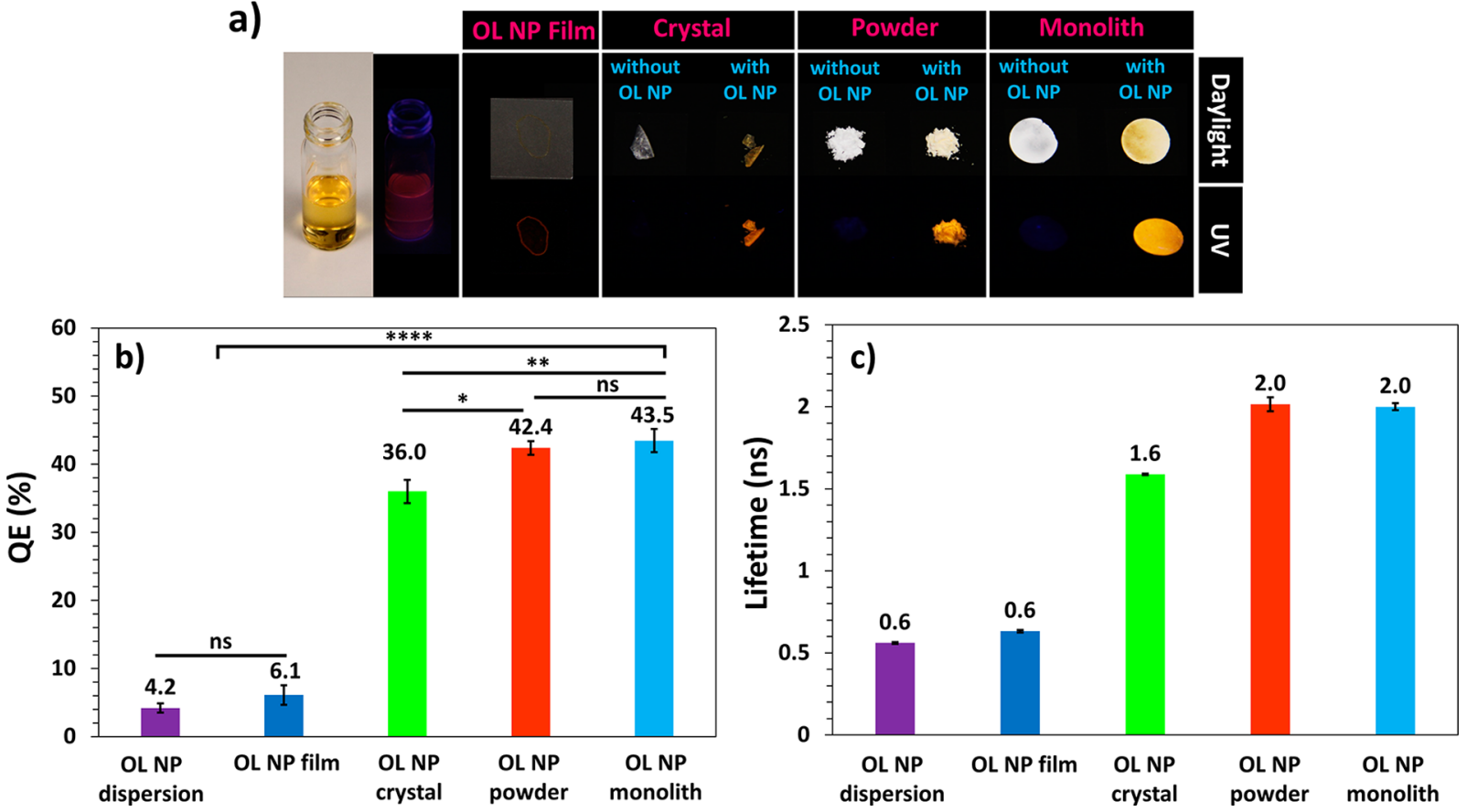

Figure 3. (a) Real-color photographs of OL NPs in all forms along with sucrose crystals, powders, and monoliths prepared without OL NPs under daylight and UV light excited at $365 \mathrm{~nm}$. (b) Quantum efficiency (ns: nonsignificant) and (c) lifetime of OL NPs in dispersion, solid film, crystal, powder, and monolith form.

concept LED employing OL NP monoliths as color converters on a blue LED chip. Considering the simplicity of our methodology along with the significantly improved QY, temperature stability, and low cost of the OL NP monoliths presented here, this approach makes the ubiquitous use of OL NPs achievable for solid-state lighting and possibly other photonic applications.

\section{RESULTS AND DISCUSSION}

Oligomer nanoparticles were prepared according to a recently reported procedure. ${ }^{18}$ To investigate the effect of the incorporated conjugated OL NPs amount on QY, four different OL NPs embedded sucrose crystals were prepared by mixing the varying volumes $(1,2,3$, and $4 \mathrm{~mL})$ of $1.3 \mathrm{mM}$ aqueous dispersion of OL NPs with $2 \mathrm{~mL}$ of saturated sucrose solution (1.5 M). Crystals were allowed to grow slowly at room temperature over $10 \mathrm{~d}$. Subsequently, the crystals were powdered by mechanical grinding and then transformed into their disc-shaped monoliths by applying a pressure of 5400 psi. As shown in Figure S1, the QY is maximized in the sample immobilizing $3 \mathrm{~mL}$ of OL NP in crystal, powder, and monolith form. Therefore, here we mainly focus on $3 \mathrm{~mL}$ OL NP incorporating samples with the highest efficiency throughout the manuscript (unless otherwise stated). For this sample, we calculated the concentration of the oligomer nanoparticles to be 11.8 pmol in $1 \mathrm{mg}$ of the crystals by redissolving the crystals in water and recording the absorption spectrum. The OL NP incorporating crystals were denoted as "OL NP crystal", the powders made from these crystals as "OL NP powder", and the monoliths prepared using these powders as "OL NP monoliths". The procedure of obtaining oligomer nanoparticles and the real color images of OL NP dispersion, crystal, powder and monolith are represented in Figure 1.

The photoluminescence (PL) spectra of OL in tetrahydrofuran (THF) and their drop-casted films along with the PL spectra of OL NPs in water, their films, powders, and monoliths were measured and displayed in Figure 2. The emission peak of the oligomer solution in THF (OL solution) was monitored at $576 \mathrm{~nm}$, and this peak red-shifted in solid film and in nanoparticle aqueous dispersion to $596 \mathrm{~nm}$ (Figure 2a), which we attribute to the nonradiative interaction between oligomer molecules in close proximity. When we compare the PL spectra (Figure 2b), we observe that crystal, powder, and monolith emissions blue-shifted and became narrower compared to the OL NP dispersion and its film. Additionally, there is a more profound blue shift in powder and monolith compared to crystals.

We also measured the absorption spectra of the OL NP film, powder, and monolith. Our results presented in Figure S2 show no significant difference between the absorption capabilities of these materials except a slight increase in crystals, monoliths, and powders compared to OL NP film, which possibly stems from the increasing scattering of the excitation photons pumping OL NPs within sucrose matrix leading to improved absorption.

We next measured the QY of OL NP dispersion; its solidfilm; and crystal, powder, and monolith form of OL NP using the methodology reported in refs 25 and 27. The significance of QY differences was analyzed using a one-way ANOVA statistical test. As depicted in Figure 3b, the QYs of the OL NP dispersion and film turned out to be very similar and were calculated as $4.2 \pm 0.7 \%$ and $6.1 \pm 1.4 \%$, respectively. After the crystallization of OL NP within the sucrose, the QY of the crystal $(36.0 \pm 1.7 \%)$ surprisingly increased by $\sim 6$-fold compared to the nanoparticle film.

This significant increase in the QY clearly suggests that the sucrose molecules passivate the nonradiative transitions in the OL NPs. It is also possible that sucrose molecules separate the OL NPs, which in turn contributes to both a QY increase and blue-shifts, and narrows the emission spectrum. We also 
a)

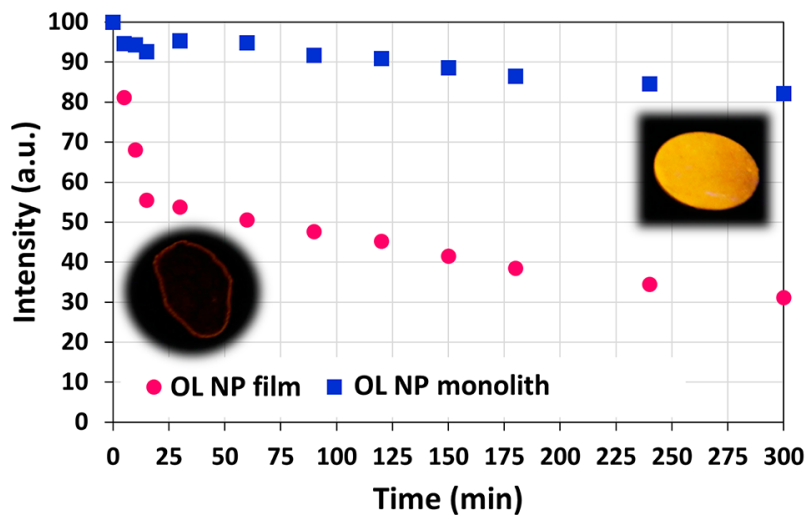

b)

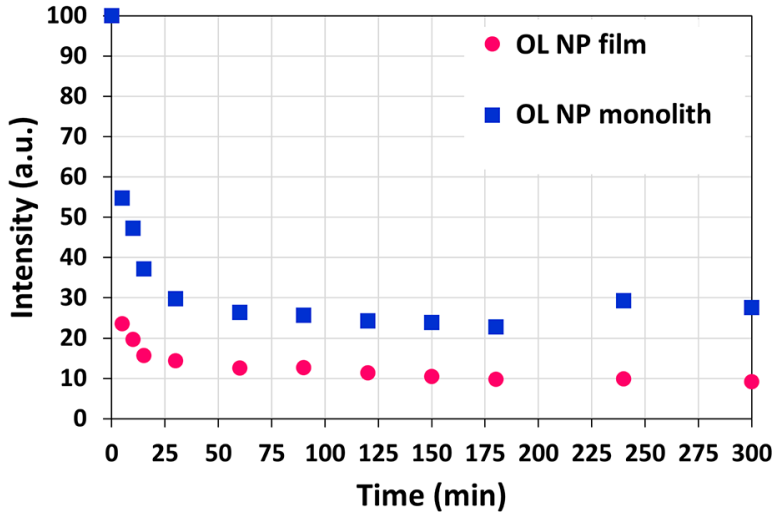

Figure 4. Normalized temperature stability of OL NP solid film (pink, circle) and OL NP monolith (blue, square) at (a) $65^{\circ} \mathrm{C}$ and $(\mathrm{b}) 130{ }^{\circ} \mathrm{C}$.

observed that powdering the crystals further increased the QY to $42.4 \pm 1.0 \%$ and the QY of the OL NP monoliths were measured to be $43.5 \pm 1.7 \%$, which is $\sim 7.5$ times that of the $\mathrm{OL}$ NP film. Since we do not expect any chemical interaction or additional passivation to occur during the powdering of crystals, we hypothesize that removal of unfavorable strain effects in the crystals contributed to the QY increase in powders as well as improved light outcoupling thanks to the scattering nature of powders. Because the roughness of the powder particles significantly decreases during the monolith formation, we expect that the scattering effects weaken and QY decreases in the monoliths. However, the QYs of powders and monoliths are still very similar, which suggests that it is mainly strain effects that influence QY increase more than scattering effect.

In our measurements, we observed that the QY increases are correlated with the blue shift of the emission peak (Figures S3 and S4) suggesting a passivation mechanism, which should also affect the fluorescence lifetime of the nanoparticles. To reveal these dynamics, we performed time-resolved fluorescence decay measurements. The calculated lifetimes presented in Figure $3 \mathrm{c}$ showed a clear increasing pattern similar to the QY measurements for all the samples indicating an inhibition of the nonradiative processes. We also performed time-resolved fluorescence decay measurements at reduced temperatures down to $50 \mathrm{~K}$ (Figure S5a). We observed that the lifetime of the OL NP film lengthens more $(40.4 \%$ change) with decreasing temperature, while the lifetime of the OL NP monolith lengthens less (23.6\% change) compared to the OL NP film. These results strongly suggest that temperature dependent nonradiative transitions such as diffusion of excitons may dominate in the OL NP film. In the OL NP monolith, however, those transitions seem to be suppressed and the nonradiative transitions with weaker temperature dependence prevail.

The QY data along with the steady-state PL spectra and time-resolved fluorescence decay measurements show that the optical features of the OL NPs are improved upon incorporation into sucrose. These changes might stem from physical or chemical interactions between the sucrose molecules and the incorporated OL NPs. To reveal the details regarding possible interactions, we compared the fluorescence lifetimes of the OL NP dispersion, OL NP in sucrose solution (without crystallization), and redissolved OL NP crystal, powder, and monolith in water. As represented in Figure $\mathrm{S} 5 \mathrm{~b}$, lifetimes of the redissolved crystal, powder, and monolith shorten and converge to the initial lifetime of OL NPs. Similarly, we observed a sharp decrease in the QY of the OL NP crystal, powder, and monolith toward the QYs of the OL NPs in the dispersion. Furthermore, we kept the OL NP sample in sucrose solution for 1 week and did not record any change in the lifetime and QY. Our attempts to detect any chemical interaction using Raman and X-ray photoelectron spectroscopies have failed due to the dominance of the sucrose signal. These findings suggest that reversible physical and/or possibly noncovalent interactions (such as hydrogen bonding) between the OL NPs and sucrose molecules may be the main causes of the observed changes in the optical features.

To understand the effect of the crystal structure on the QY of the OL NPs incorporated into sucrose, we quickly evaporated the solvent of the mixture consisting of saturated sucrose solution and the OL NP dispersion using a vacuum chamber and also using a lyophilizer (Figure S6), then powdered these highly amorphous solids, formed their monoliths, and measured their QYs. We observed that the samples prepared using these fast evaporation techniques acquire similar QYs compared with the samples prepared by slowly evaporating the water (Table S1). Therefore, we conclude that having a well-defined crystal structure does not play a significant role in achieving high QYs, while the passivation of nonradiative transitions in sucrose host may be the strongest reason for QY enhancement.

Since the improved temperature stability of the colorconverting emitters are of significant importance especially for the solid-state lighting, we studied the photoluminescence intensity change of the OL NP film and OL NP monolith at 65 and $130^{\circ} \mathrm{C}$, which are carefully chosen for lighting applications (Figure 4 and Figure S7).

These temperatures were deliberately selected because we measured the temperature of the LED chip around the location, where the monolith is placed, around $65^{\circ} \mathrm{C}$ (Figure S8) using an infrared camera. Moreover, the typical junction temperature of the InGaN/GaN-based LED chips is above $130{ }^{\circ} \mathrm{C} .{ }^{28}$ In our emission stability tests, we heated the samples on a hot plate at these temperatures without interruption and recorded their PL intensities at different time points. As shown in Figure $4 \mathrm{a}$, the OL NP film lost $45 \%$ of its initial PL intensity within $25 \mathrm{~min}$ at $65{ }^{\circ} \mathrm{C}$; on the other hand, the OL NP monolith lost only $7 \%$ of its PL intensity at the same time interval. After $5 \mathrm{~h}$ of exposure, OL NP film lost $69 \%$ of its PL intensity, whereas the monolith lost just $18 \%$ of its PL. The pattern was similar for the samples heated at $130{ }^{\circ} \mathrm{C}$ (Figure $4 \mathrm{~b}$ ). After a rapid decrease in the PL 

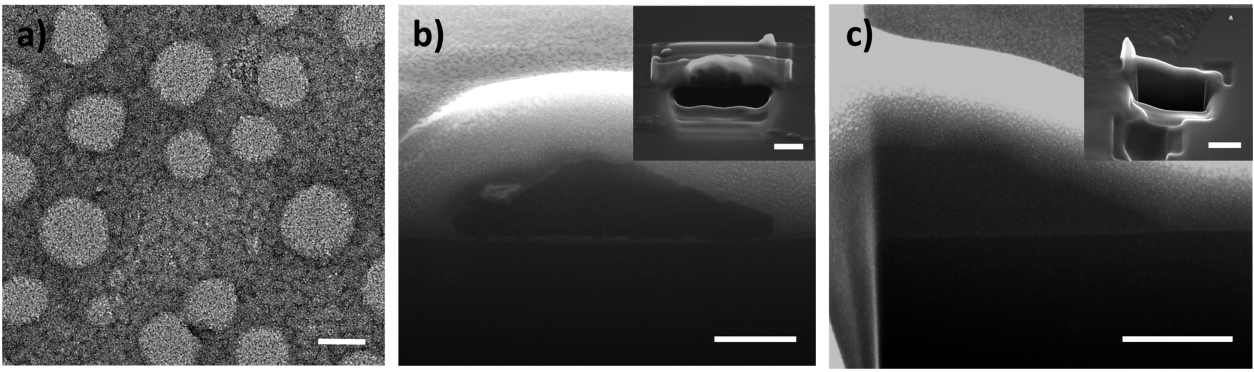

Figure 5. (a) Transmission electron microscopy image of OL NPs (scale bar: $50 \mathrm{~nm}$ ). Scanning electron microscopy image of (b) a single large OL NP (>500 nm) and (c) an aggregated area consisting of OL NPs with the diameter of $98 \mathrm{~nm}$ (scale bars: $200 \mathrm{~nm}$ ). Insets show the zoom-out of the imaged area (scale bars: $500 \mathrm{~nm}$ ).

intensity, the decrease slowed down, and at the end of the test, OL NP film and OL NP monolith lost $91 \%$ and $72 \%$ of their initial PL intensities, respectively. According to these results, we conclude that OL NP monoliths showed improved stability compared to the OL NP film at both temperatures indicating the suitability of this material system for solid-state lighting applications.

Subsequent to optical characterizations, we carried out the morphological characterizations starting with the transmission electron microscopy (TEM) imaging of OL NPs. Contrast difference was provided by negative staining of the TEM sample and the images are presented in Figure 5a. As depicted in this figure, OL NPs exhibited well-dispersed spherical shapes with sizes of $56.6 \pm 7.7 \mathrm{~nm}$, which is also verified by dynamic light scattering (DLS) measurements to be $57.17 \mathrm{~nm}$.

We also imaged the nanoparticles by using scanning electron microscopy (SEM) after etching some portion of the particle with focused-ion beam (FIB) (Figure 5b,c). We first etched nanoparticles with the size of $56.6 \mathrm{~nm}$, which are used in the crystallization processes. However, FIB exposure etched the whole nanoparticle due to the small size that they have; therefore, we needed larger nanoparticles. For this purpose, we used two larger OL NPs with the sizes of $>500$ and $98 \mathrm{~nm}$ for imaging. Figure $5 \mathrm{~b}$ shows the SEM image of OL NP $(>500$ $\mathrm{nm}$ ) on a silicon wafer, which was etched in half using FIB. We subsequently imaged smaller OL NPs (with the diameter of 98 $\mathrm{nm}$ ) to be more correlated with our experiments (Figure 5c). In this case, however, the small sized nanoparticles did not survive after FIB etching. Therefore, we took images from an aggregated area (Figure 5c, inset) where we observed flu filamentous structures with very low contrast in the center of the particle indicating the micelle-like structure of the nanoparticle. We believe that these structures allow for the penetration of the sucrose molecules between the oligomer molecules and help in the passivation of the nonradiative transitions during the crystallization, which in turn helps increasing the QY. In addition to these images, we also attempted to take the TEM images of the OL NPs in the sucrose matrix by following the technique presented in ref 29; however, no contrast could be observed between the organic nanoparticles and the host medium.

After the nanoparticles were imaged, we further characterized the crystal structure of OL NP crystals, OL NP powders, and the OL NP monoliths using X-ray diffraction spectroscopy (XRD) (Figure S9). The crystal structure of sucrose was identified as monoclinic (JCPDS\#24-1977). As shown in Figure S9, OL NP crystals have prominent peaks at $11.6^{\circ}, 16.7^{\circ}, 22.5^{\circ}$, $25.2^{\circ}$, and $38.3^{\circ}$, which are in line with the literature showing the crystal structure of sucrose. ${ }^{29} \mathrm{We}$ found out that powdering the crystals increases the polycrystalline character with dominant XRD signal occurring at $22.5^{\circ}$. Furthermore, no significant change in the XRD signal was observed after the formation of monoliths under high pressure. Thus, we conclude that this level of pressure expectedly did not change the crystal structure of OL NP powders in parallel with our observations of insignificant $\mathrm{QY}$ and lifetime changes upon monolith formation.

Considering the low QYs of the organic nanoparticle solid films, especially at high particle densities required for solid-state lighting applications, the methodology that we present here makes these nanoparticles suitable color converters for solidstate lighting. With this motivation, we employed these OL NP monoliths as color converters on a high power blue LED emitting at $460 \mathrm{~nm}$ to obtain a proof-of-concept LED. The spectrum of the resulting OL NP monolith integrated LED is presented in Figure 6.

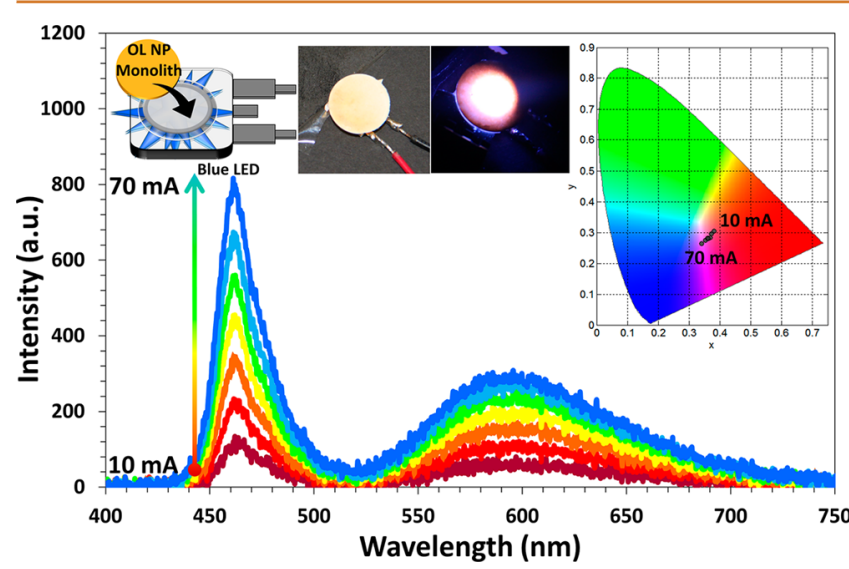

Figure 6. Emission spectrum of the OL NP monolith integrated blue LED at increasing currents along with the chromaticity points of the emitted light (inset, right). Moreover, representative image, real color images of OL NP monolith on LED without current flow and with current flow are presented from left to right (inset, left).

On the basis of this data, we calculated the chromaticity coordinates and correlated color temperatures (CCTs) of the emission to characterize the shade of the white emission. Results show that the generated white light has a warm white color up to $80 \mathrm{~mA}$ as indicated by the color coordinates around $(0.37,0.29)$ and CCTs $\sim 3200-3700 \mathrm{~K}$ (Figure S10). After this current level, however, the emission from the blue LED chip becomes significantly strong making the appearance of the emitted light bluish as also shown by the chromaticity coordinates around $(0.20,0.15)$ and CCTs of $\sim 30000 \mathrm{~K}$. 
We also evaluated the color rendition performance of the LED by calculating the color quality scale (CQS) (Figure S11). The resulting CQS values around 70 at low current ranges indicate that this white LED exhibits mediocre color rendition capability. We later calculated the luminous efficiency of the LED to be in the range of $15-20 \mathrm{~lm} / \mathrm{W}_{\text {elect }}$. The luminous efficacy of optical radiation (LER) values of this LED escalate around $250 \mathrm{~lm} / \mathrm{W}_{\text {opt }}$ at low current levels indicating a small overlap of the emission spectrum with the human eye sensitivity function. This LER performance, however, can be improved if the narrow emitting nanoparticles with PL peaks close to $550 \mathrm{~nm}$ are employed.

\section{CONCLUSION}

In this study, we report the incorporation of oligomer nanoparticles into an organic host to obtain highly efficient and highly stable fully organic color-converting monoliths suitable for solid-state lighting. In this presented approach, sucrose is used to wrap oligomer nanoparticles, and the powders of their crystals are employed to form monolith. The fluorescence quantum yields of the resulting solid monoliths reached $43.5 \%$, while those of their direct solid film remained only at $6.1 \%$. Furthermore, we showed that these oligomer nanoparticle monoliths improve the temperature stability of the oligomer nanoparticle emission by $\sim 3$-fold compared to the bare oligomer nanoparticle solid-film. We believe that the considerably increased quantum yield and temperature stability of the oligomer emission within monoliths, in addition to the low cost and simplicity of the preparation method presented here, will provide opportunities to deploy oligomer nanoparticles in solid-state lighting and possibly other photonic applications.

\section{EXPERIMENTAL SECTION}

Chemicals. All chemicals and solvents to synthesize OL and OL NPs were purchased from Sigma-Aldrich Chemical Co. (Germany). Sucrose was also obtained from Sigma-Aldrich Chemical Co.

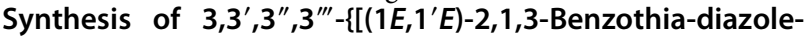
4,7-diylbis(ethene-2,1-diyl)]-bis(9H-fluorene-9,9,2-triyl)\}tetrakis( $N, N$-dimethylpropan-1-amine) Oligomer Nanoparticles. Before OL NP preparation, oligomer was synthesized according to the procedure reported by Pennakalathil et al. ${ }^{18}$ To convert the OL into nanoparticles, $10 \mathrm{mg}$ of oligomer was dissolved in $10 \mathrm{~mL}$ of THF and the resulting solution was filtered through $0.45 \mu \mathrm{m}$ syringe filter. One milliliter of this OL solution was injected into $20 \mathrm{~mL}$ of deionized water slowly under sonication. After $30 \mathrm{~min}$ sonication, THF was removed under reduced pressure at room temperature via rotary evaporator.

Dynamic Light Scattering (DLS) Measurements. Hydrodynamic size of OL NPs was measured using Malvern Nano-ZS Zetasizer. Size distribution of number was reported as the average diameter.

Preparation of Crystals, Monoliths, and Films. Sucrose stock solution used in this study was prepared by dissolving sucrose $(260 \mathrm{~g})$ in $500 \mathrm{~mL}$ of Milli-Q water. For the crystallization, 1, 2, 3, and $4 \mathrm{~mL}$ of OL NP solution $(0.11 \mathrm{mg} / \mathrm{mL})$ was mixed with the $2 \mathrm{~mL}$ of saturated sucrose stock solution. Water of the mixtures was evaporated on a vibration minimizing pad purchased from Sorbothane at room temperature. After a few days of drying, formation of macrocrystals was completed (OL NP crystals).

To obtain OL NP powders, these crystals were mechanically powdered using a mortar. OL NP monoliths for all concentrations were prepared by applying pressure. Briefly, $\sim 80 \mathrm{mg}$ of OL NP powders was placed between two stainless steel disks and a pressure of $5400 \mathrm{psi}$ was applied using a hydraulic press for $5 \mathrm{~min}$ at room temperature. The thickness and size of the monoliths were measured as 0.5 and $13.0 \mathrm{~mm}$, respectively. For comparison, films of OL solution in THF and OL NP solution in double distilled water $\left(\mathrm{ddH}_{2} \mathrm{O}\right)$ were prepared by dropping $100 \mu \mathrm{L}$ of these solutions onto a glass substrate and dried at room temperature.

Transmission Electron Microscopy (TEM). Morphological structure of the OL NPs was investigated using a FEI Tecnai G2 F30 transmission electron microscope. Sample for imaging was prepared by dropping $2 \mu \mathrm{L}$ of stock OL NP solution on a 200 mesh copper grid and staining with uranyl acetate $(2 \%, \mathrm{w} / \mathrm{v})$ for 1 min.

Focused Ion Beam Scannıng Electron Microscopy (FIB-SEM). Oligomer nanoparticle cross-sectional images were taken using FEI NanoSEM dual beam system. OL NPs with the different diameters were spin coated onto a silicon wafer. Prior to imaging, protective platinum layer was coated with electron beam and ion beam, respectively. Careful ion etching was performed at low ion currents, and cross-sectional images were obtained after etching.

X-ray Diffraction Spectroscopy (XRD). Crystal structures of OL NP crystal, powder, and monolith were investigated using a Panalytical X'pert Pro Multipurpose X-ray Diffractometer operated at $45 \mathrm{kV}$ and $20 \mathrm{~mA}$ with $\mathrm{Cu} \mathrm{K} \alpha$ radiation and a diffracted beam monochromator. XRD spectra were obtained between $10^{\circ}$ and $60^{\circ}$ of the $2 \theta$ angle with a step size of $0.01^{\circ}$.

Photoluminescence (PL) and Quantum Yield (QY) Measurements. PL spectra of varying forms of OL and OL NPs were taken using an Ocean Optics Maya 2000 spectrometer equipped with an integrating sphere, a xenon lamp, and a monochromator at an excitation wavelength of $400 \mathrm{~nm}$. QY of solutions, films, and OL NPs in crystal, powder, and monolith forms were obtained using the method described elsewhere. ${ }^{25,27}$ Statistical analyses were performed using GraphPad Prism 5, and one-way ANOVA and Tukey's multiple comparisons post-tests were employed to determine the statistical difference in quantum efficiencies.

Lifetime and Temperature Dependent Lifetime Measurements. Time-resolved fluorescence decays of the OL NP dispersion, drop-casted film, crystals, powders, and monoliths were acquired by using a PicoHarp 200 time-resolved single photon counting system (PicoQuant). The time-resolved photon count decay curves were recorded at the wavelengths where the emission maxima were observed in the PL measurements. Amplitude-averaged lifetimes were extracted by exponential reconvolution method using the FluoFit software. Temperature dependent lifetime of OL NP film and OL NP monolith were measured at 50,100,150, 200, 250, and $300 \mathrm{~K}$ using the same setup.

LED Preparation. The OL NP monoliths were directly placed on an Avago ASMT blue LED. The luminance was recorded under varying current flows between 10 and $350 \mathrm{~mA}$ using an Ocean Optics integrating sphere and spectrometer. Colorimetric and photometric characterizations were carried out employing in-house written codes.

\section{ASSOCIATED CONTENT}

\section{Supporting Information}

The Supporting Information is available free of charge on the ACS Publications website at DOI: 10.1021/acsnano.6b01473.

QY of OL NP dispersion and film along with OL NP powder, crystal, and monolith incorporating various amounts of OL NPs; absorption spectra of OL NP film, crystal, powder, and monolith; PL peaks and QYs of oligomer in THF, oligomer NPs in water, and their dropcasted films together with the OL NP crystals, powders, and monoliths as a function of OL NP incorporation amount; temperature dependent lifetime of OL NP drop-casted film and monolith, lifetime of redissolved OL NP crystal, powder and monolith along with OL NP dispersion in water and OL NP dispersion in sucrose without crystallization; real-color photographs of quickly evaporated OL NP and saturated sucrose mixtures, their powders, and monoliths; PL spectra of the drop-casted 
OL NP film and monolith at 65 and $130{ }^{\circ} \mathrm{C}$; thermal camera image of the LED; XRD spectra of OL NP crystal, monolith, and powder; and the photometric performance of the LED (PDF)

\section{AUTHOR INFORMATION}

\section{Corresponding Author}

*E-mail: volkan@bilkent.edu.tr.

\section{Author Contributions}

${ }^{\ddagger}$ Z.S.-E. and T.E. contributed equally.

\section{Notes}

The authors declare no competing financial interest.

\section{ACKNOWLEDGMENTS}

We acknowledge ESF EURYI, EU-FP7 Nanophotonics4Energy NoE, BMBF TUR 09/001, and TUBITAK EEEAG 109E002, 109E004, 110E010, 110E217, and 112E183, in part by NRFCRP-6-2010-02 and NRF-RF-2009-09. H.V. Demir acknowledges TUBA-GEBIP and T. Erdem acknowledges TUBITAK BIDEB. We thank $\mathrm{H}$. Keita for the preparation of conjugated oligomer nanoparticles.

\section{REFERENCES}

(1) AlSalhi, M. S.; Alam, J.; Dass, L. A.; Raja, M. Recent Advances in Conjugated Polymers for Light Emitting Devices. Int. J. Mol. Sci. 2011, 12, 2036-2054.

(2) Park, E.-J.; Erdem, T.; Ibrahimova, V.; Nizamoglu, S.; Demir, H. V.; Tuncel, D. White-Emitting Conjugated Polymer Nanoparticles with Cross-Linked Shell for Mechanical Stability and Controllable Photometric Properties in Color-Conversion LED Applications. ACS Nano 2011, 5, 2483-2492.

(3) Jiang, C.; Yang, W.; Peng, J.; Xiao, S.; Cao, Y. High-Efficiency, Saturated Red-Phosphorescent Polymer Light-Emitting Diodes Based on Conjugated and Non-Conjugated Polymers Doped with an Ir Complex. Adv. Mater. 2004, 16, 537-541.

(4) Kuik, M.; Wetzelaer, G.-J. A. H.; Nicolai, H. T.; Craciun, N. I.; De Leeuw, D. M.; Blom, P. W. M. 25th Anniversary Article: Charge Transport and Recombination in Polymer Light-Emitting Diodes. Adv. Mater. 2014, 26, 512-531.

(5) Tuncel, D.; Demir, H. V. Conjugated Polymer Nanoparticles. Nanoscale 2010, 2, 484.

(6) Beek, W. J. E.; Wienk, M. M.; Janssen, R. A. J. Efficient Hybrid Solar Cells from Zinc Oxide Nanoparticles and a Conjugated Polymer. Adv. Mater. 2004, 16, 1009.

(7) Li, G.; Zhu, R.; Yang, Y. Polymer Solar Cells. Nat. Photonics 2012, $6,153-161$

(8) Liang, Y.; Xu, Z.; Xia, J.; Tsai, S. T.; Wu, Y.; Li, G.; Ray, C.; Yu, L. For the Bright Future-Bulk Heterojunction Polymer Solar Cells with Power Conversion Efficiency of 7.4\%. Adv. Mater. 2010, 22, E135E138.

(9) Liu, H.-Y.; Wu, P.-J.; Kuo, S.-Y.; Chen, C.-P.; Chang, E.-H.; Wu, C.-Y.; Chan, Y.-H. Quinoxaline-Based Polymer Dots with Ultrabright Red to Near-Infrared Fluorescence for In Vivo Biological Imaging. J. Am. Chem. Soc. 2015, 137, 10420-10429.

(10) Wu, C.; Bull, B.; Szymanski, C.; Christensen, K.; McNeill, J. Multicolor Conjugated Polymer Dots for Biological Fluorescence Imaging. ACS Nano 2008, 2, 2415-2423.

(11) Wu, C.; Szymanski, C.; McNeill, J. Preparation and Encapsulation of Highly Fluorescent Conjugated Polymer Nanoparticles. Langmuir 2006, 22, 2956-2960.

(12) Park, Y.; Advincula, R. C. Hybrid Semiconductor Nanoparticles: $\pi$-Conjugated Ligands and Nanostructured Films. Chem. Mater. 2011, 23, 4273-4294.

(13) Wu, X.; Li, H.; Xu, Y.; Xu, B.; Tong, H.; Wang, L. Thin Film Fabricated from Solution-Dispersible Porous Hyperbranched Con- jugated Polymer Nanoparticles without Surfactants. Nanoscale 2014, 6, 2375-2380.

(14) Huyal, I. O.; Ozel, T.; Tuncel, D.; Demir, H. V. Quantum Efficiency Enhancement in Film by Making Nanoparticles of Polyfluorene. Opt. Express 2008, 16, 13391-13397.

(15) Haugeneder, A.; Lemmer, U.; Scherf, U. Exciton Dissociation Dynamics in a Conjugated Polymer Containing Aggregate States. Chem. Phys. Lett. 2002, 351, 354-358.

(16) Gierschner, J.; Egelhaaf, H.-J.; Oelkrug, D.; Müllen, K. Electronic Deactivation and Energy Transfer in Doped Oligophenylenevinylene Nanoparticles. J. Fluoresc. 1998, 8, 37-44.

(17) Martini, I.; Smith, A.; Schwartz, B. Exciton-Exciton Annihilation and the Production of Interchain Species in Conjugated Polymer Films: Comparing the Ultrafast Stimulated Emission and Photoluminescence Dynamics of MEH-PPV. Phys. Rev. B: Condens. Matter Mater. Phys. 2004, 69, 035204.

(18) Pennakalathil, J.; Jahja, E.; Ozdemir, E. S.; Konu, O.; Tuncel, D. Red Emitting, Cucurbituril-Capped, pH-Responsive Conjugated Oligomer-Based Nanoparticles for Drug Delivery and Cellular Imaging. Biomacromolecules 2014, 15, 3366-3374.

(19) Fischer, I.; Kaeser, A.; Peters-Gumbs, M. a M.; Schenning, A. P. H. J. Fluorescent $\pi$-Conjugated Polymer Dots versus Self-Assembled Small-Molecule Nanoparticles: What's the Difference? Chem. - Eur. J. 2013, 19, 10928-10934.

(20) Dang, Z.-M.; Gao, Y.; Xu, H.-P.; Bai, J. Fabrication and Characteristics of Organic Semiconductor Nanoparticles of Copper Phthalocyanine Oligomers. J. Colloid Interface Sci. 2008, 322, 491-496.

(21) Liu, X.; He, X.; Jiu, T.; Yuan, M.; Xu, J.; Lv, J.; Liu, H.; Li, Y. Controlled Aggregation of Functionalized Gold Nanoparticles with a Novel Conjugated Oligomer. ChemPhysChem 2007, 8, 906-912.

(22) Otto, T.; Müller, M.; Mundra, P.; Lesnyak, V.; Demir, H. V.; Gaponik, N.; Eychmüller, A. Colloidal Nanocrystals Embedded in Macrocrystals: Robustness, Photostability, and Color Purity. Nano Lett. 2012, 12, 5348-5354.

(23) Kalytchuk, S.; Zhovtiuk, O.; Rogach, A. L. Sodium Chloride Protected CdTe Quantum Dot Based Solid-State Luminophores with High Color Quality and Fluorescence Efficiency. Appl. Phys. Lett. 2013, 103, 103105.

(24) Müller, M.; Kaiser, M.; Stachowski, G. M.; Resch-Genger, U.; Gaponik, N.; Eychmüller, A. Photoluminescence Quantum Yield and Matrix-Induced Luminescence Enhancement of Colloidal Quantum Dots Embedded in Ionic Crystals. Chem. Mater. 2014, 26, 3231-3237.

(25) Erdem, T.; Soran-Erdem, Z.; Sharma, V. K.; Kelestemur, Y.; Adam, M.; Gaponik, N.; Demir, H. V. Stable and Efficient Colour Enrichment Powders of Nonpolar Nanocrystals in LiCl. Nanoscale 2015, 7, 17611-17616.

(26) Adam, M.; Erdem, T.; Stachowski, G. M.; Soran-Erdem, Z.; Lox, J.; Bauer, C.; Poppe, J.; Demir, H. V.; Gaponik, N.; Eychmueller, A. Implementation of High-Quality Warm-White Light-Emitting Diodes by a Model-Experimental Feedback Approach Using Quantum DotSalt Mixed Crystals. ACS Appl. Mater. Interfaces 2015, 7, 2336423371.

(27) de Mello, J. C.; Wittmann, H. F.; Friend, R. H. An Improved Experimental Determination of External Photoluminescence Quantum Efficiency. Adv. Mater. 1997, 9, 230.

(28) OSRAM OptoSemiconductors. LED Fundamentals. Thermal Characteristics of LEDs http://ledlight.osram-os.com/wp-content/ uploads/2013/01/OSRAM-OS LED-FUNDAMENTALS ThermalCharacteristics-of-LEDs_v2_08-16-11_SCRIPT.pdf (accessed Jan 7, 2016).

(29) Erdem, T.; Soran-Erdem, Z.; Hernandez-Martinez, P. L.; Sharma, V. K.; Akcali, H.; Akcali, I.; Gaponik, N.; Eychmüller, A.; Demir, H. V. Sweet Plasmonics: Sucrose Macrocrystals of Metal Nanoparticles. Nano Res. 2015, 8, 860-869. 